\title{
Sound and light in turbid media
}

\section{Lihong V. Wang, Geng Ku, Qimin Shen}

Lihong V. Wang, Geng Ku, Qimin Shen, "Sound and light in turbid media," Proc. SPIE 3601, Laser-Tissue Interaction X: Photochemical, Photothermal, and Photomechanical, (14 June 1999); doi: 10.1117/12.350005

Event: BiOS '99 International Biomedical Optics Symposium, 1999, San Jose, CA, United States 


\title{
Sound and Light in Turbid Media
}

\author{
Lihong V. Wang, Geng Ku, and Qimin Shen \\ Optical Imaging Laboratory \\ Biomedical Engineering Program \\ Texas A\&M University \\ College Station, Texas 77843-3120, USA
}

\begin{abstract}
Two imaging techniques combining ultrasound and light are reviewed. The motivation is to combine the advantages of optical information and acoustic imaging resolution. The first technique is sonoluminescence tomography, where a sonoluminescence signal generated internally in the media by continuous-wave ultrasound is used. Twc-dimensional images can be produced for objects embedded in turbid media by raster scanning the media. The second technique is ultrasoundmodulated optical tomography, where a frequency-swept ultrasonic wave was used to modulate the laser light passing through a scattering medium. Multiple 1D images obtained at various positions perpendicular to the ultrasonic axis were composed to obtain a 2D tomographic image of the medium.
\end{abstract}

Keywords: Sonoluminescence, tomography, scattering media, ultrasound, ultrasound-modulation

\section{INTRODUCTION}

Optical imaging, also known as optical tomography, in strongly scattering media, has become an active research field because of its advantages of noninvasion, nonionization, and functional contrast for biomedical diagnosis. ${ }^{1,2}$ Several optical imaging techniques being investigated include time-resolved optical imaging, frequency-domain optical imaging, optical coherence tomography, optoacoustic tomography and ultrasound-modulated optical (acousto-optical) tomography. In these approaches, time-resolved and frequency-domain techniques have achieved a comparable resolution of millimeters. Optical coherence tomography has achieved $10-\mu \mathrm{m}$ resolution but is limited to a penetration of $<2$ millimeters into biological tissues. Optoacoustic tomography and acousto-optical tomography have achieved millimeter resolution and have potential to image thick biological tissues. The image contrast is based on the difference in optical properties between abnormal and the surrounding normal biological tissues. All these optical approaches use an external light source, mostly a laser.

Because biological tissues are optically turbid media, light is quickly diffused in tissues due to strong scattering. Light transmitted through tissues consists of three types: ballistic light, quasi-ballistic light, and diffuse light. Ballistic light travels straight through tissue with no experience of scattering by the tissue and hence carries direct imaging information as $\mathrm{x}$-ray does. Quasi-ballistic light experiences minimal scattering in the forward direction and carries some imaging information. Diffuse light follows tortuous paths, carries little direct imaging information and overshadows ballistic or quasiballistic light. For a 5-cm-thick biological tissue with the assumed absorption coefficient $\mu_{\mathrm{a}}=0.1 \mathrm{~cm}^{-1}$ and reduced scattering coefficient $\mu_{\mathrm{s}}^{\prime}=10 \mathrm{~cm}^{-1}$, the ballistic light and quasi-ballistic light does not exist for practical purposes. ${ }^{3}$ Therefore, diffuse light is the only carrier of imaging information for thick biological tissues. All optical tomography for thick biological tissues must overcome the light scattering problem to obtain optical images.

\section{SONOLUMINESCENCE TOMOGRAPHY}

Ultrasonic generation of light in a medium, known as sonoluminescence (SL), was first discovered in $1934 .^{4}$ The initial observations were multiple-bubble sonoluminescence (MBSL). SL has attracted an extraordinary amount of attention in this decade since single-bubble sonoluminescence (SBSL) was reported in $1990 .^{5-13}$ Although the full explanation of SL is still in development, it is well known that light is emitted when tiny bubbles driven by ultrasound collapse. The bubbles start out with a radius of several microns and expand to $\sim 50$ microns due to a decrease in acoustic pressure in the negative half of a sinusoidal period. After the sound wave reaches the positive half of the period, the situation rapidly changes. The resulting 
pressure difference leads to a rapid collapse of the bubbles accompanied by a broadband emission of light sonoluminescence. This process repeats with each cycle of sound. ${ }^{13}$ The flash time of SL was measured to be in the tens of picoseconds. ${ }^{5}$ SBSL is so bright that it can be seen by the naked eye even in a lighted room, whereas MBSL is visible only in a darkened room. ${ }^{10}$ The spectrum of SL contains molecular emission bands associated with the liquid, mostly water, in which the sonoluminescence occurs. ${ }^{10}$ A typical spectrum of SL is a broadband emission with peaks near $300-500 \mathrm{~nm} .{ }^{13}$ There are also spectral peaks reported at $590 \mathrm{~nm}, 670 \mathrm{~nm}$ and $770 \mathrm{~nm}$ while alkali-metal salt of $\mathrm{Na}^{+}, \mathrm{Li}^{+}$and $\mathrm{K}^{+}$were dissolved in water, respectively. ${ }^{6}$ From the spectrum of SL in water, the local temperature within the cavities was estimated on the order of $5000 \mathrm{~K} .{ }^{10}$ Researchers have envisaged possible applications of SL in sonofusion, sonochemistry, and building ultrafast lasers using the ultrafast flash of light in SL, which is the only means of generating picosecond flashes of light besides a laser.

We report here a comprehensive study of a novel application of sonoluminescence in optical imaging: sonoluminescent tomography (SLT) of dense turbid media. ${ }^{14}$ In SLT, minimally scattering ultrasound that generates an internal light source in the media is used to image optically scattering media. SLT contains information not available in the traditional ultrasonography. The major advantages of SLT include: 1) high signal-noise ratio (SNR) due to the internally generated SL signal; 2) high contrast of imaging; 3) good spatial resolution, which is limited by the ultrasonic focal size; and 4) low cost of equipment. The cost of equipment to generate SL is as low as hundreds of U.S. dollars. ${ }^{15}$ This paper gives a full account of our investigation while our previous Letter demonstrated the concept of SLT. ${ }^{14}$

An Intralipid phantom was prepared by mixing $8 \mathrm{ml}$ of dominantly scattering Intralipid (Pharmacia Inc., 20\%) and $3.25 \times 10^{-8} \mathrm{~mol}$ of dominantly absorbing Trypan Blue dye (Sigma, T5526) in $360 \mathrm{ml}$ of distilled water. The reduced scattering coefficient $\mu_{\mathrm{s}}^{\prime}$ and the absorption coefficient $\mu_{\mathrm{a}}$ were respectively $6.15 \mathrm{~cm}^{-1}$ and $0.014 \mathrm{~cm}^{-1}$ at the wavelength of $584 \mathrm{~nm}$, which is the absorption peak of Trypan Blue dye. The reduced scattering coefficient $\mu_{\mathrm{s}}$ ' is equal to $\mu_{\mathrm{s}} \times(1-\mathrm{g})$, where $\mu_{\mathrm{s}}$ is the scattering coefficient defined as the probability of scattering per unit infinitesimal path length, and $g$ is the scattering anisotropy defined as the average cosine of the single-scattering deflection angle. Since the spectrum of SL is broadband, ${ }^{16}$ the optical scattering properties of the scattering media were also given at the wavelength of $400 \mathrm{~nm}$, corresponding to the spectral peaks of the $\mathrm{SL}^{17}$ and the maximum-sensitivity spectral range of the optical detector in our experiment. The $\mu_{\mathrm{s}}{ }^{\prime}$ and $\mu_{\mathrm{a}}$ of the Intralipid phantom at $400 \mathrm{~nm}$ were $8.5 \mathrm{~cm}^{-1}$ and $0.002 \mathrm{~cm}^{-1}$, respectively. ${ }^{18}$

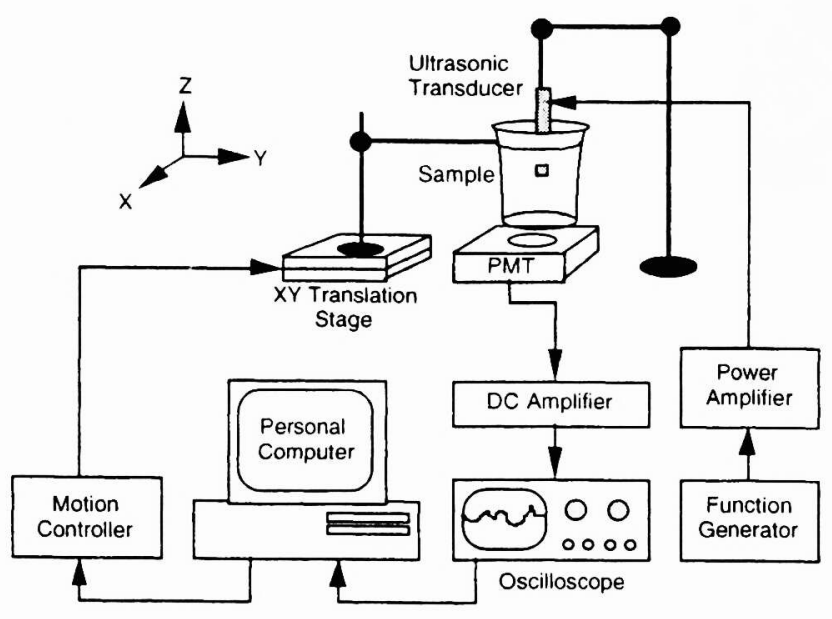

Figure 1. Schematic diagram of the experimental setup for sonoluminescence imaging.

The turbid solution was contained in a 400-ml fused-quartz beaker (Quartz Scientific Inc., QBKLO400) and was held on an $x-y$ translation stage (Figure 1). An objects made of rubber was buried in the phantom. An ultrasonic transducer (Panametrics, V314-SU) with a focal length of $3.68 \mathrm{~cm}$, a focal diameter of $0.3 \mathrm{~cm}$, and a focal zone of $3.44 \mathrm{~cm}$ transmitted vertically an ultrasonic wave into the scattering medium. The ultrasonic transducer was driven by an amplified 1-MHz sinusoidal signal from a function generator (Stanford Research System, DS345). The amplification was achieved by use of a power amplifier (Mini-Circuits, TIA-1000-1R8) and a transformer. The height of the ultrasonic transducer was adjusted such that the focal zone of the ultrasonic wave enclosed the buried object in the vertical direction. The height between the middle plane of the object and the bottom of the beaker was $4.5 \mathrm{~cm}$. The motorized translation stage, controlled by a personal computer (PC), was able to scan along both the $x$ and the $y$ axes, which formed an $x-y$ plane perpendicular to the ultrasonic 
axis. The SL signal was detected by a photomultiplier tube (PMT) (Hamamatsu, R928) beneath the beaker, and then was differentially amplified by a low-noise preamplifier (Stanford Research System, SR560). The amplified signal was a dc voltage representing a time-averaged SL intensity with a time constant of $\sim 10 \mathrm{~ms}$ for the detection system. The amplified signal was recorded by a digital oscilloscope (Tektronix, TDS 640A) and was subsequently acquired by the PC through a GPIB interface (National Instruments, PCI-GPIB).

While raster scanning the beaker in the $x-y$ plane with a step size of $1 \mathrm{~mm}$, the PC recorded the dc signals of SL versus the values of $x$ and $y$. The optical and ultrasonic systems were fixed while the beaker was scanned. Two-dimensional images of the objects buried in the scattering media were plotted with the acquired data.

We previously reported the SL column in a clear solution that was imaged with a CCD camera [Figure $2(a)] .{ }^{14}$ We further modeled the distribution of acoustic pressure underneath the ultrasonic transducer using the following equation.

$$
p(\boldsymbol{r}, t)=C \int_{s} \frac{p(s) \exp [j(\omega t-\phi)]}{d} d s,
$$

where $p(r, t)$ is the pressure as a function of the observation point $r$ and time $t, C$ is a constant, $p(s)$ is the pressure on the surface of the ultrasonic transducer, $\omega$ is the angular frequency of the ultrasonic wave, $\phi$ is the phase delay from a point on the transducer surface and the point of observation, and $d$ is the distance between a point on the transducer surface and the point of observation. The integration is over the surface of the ultrasonic transducer. The focal length in the calculated sound field matched that specified by the manufacturer [Figure 2(b)]. The sound column strongly correlated with the SL column.

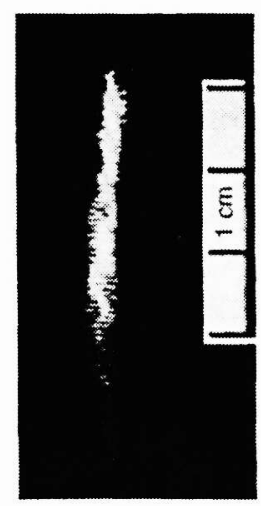

(a)
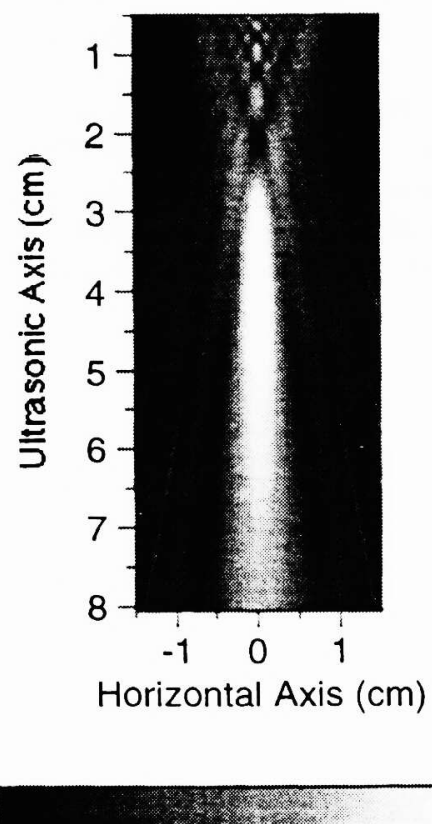

$\begin{array}{lllllll}0.0 & 0.1 & 0.2 & 0.3 & 0.4 & 0.5 & 0.6\end{array}$ Acoustic Pressure (a.u.)

(b)

Figure 2. (a) Sonoluminescence column measured with a CCD camera. (b) Modeled sound column of the ultrasonic transducer.

A rubber cube in the Intralipid phantom was imaged by SLT (Figure 3). The spatial resolution of the edges was estimated to be $2-3 \mathrm{~mm}$, and an excellent imaging contrast was observed. 

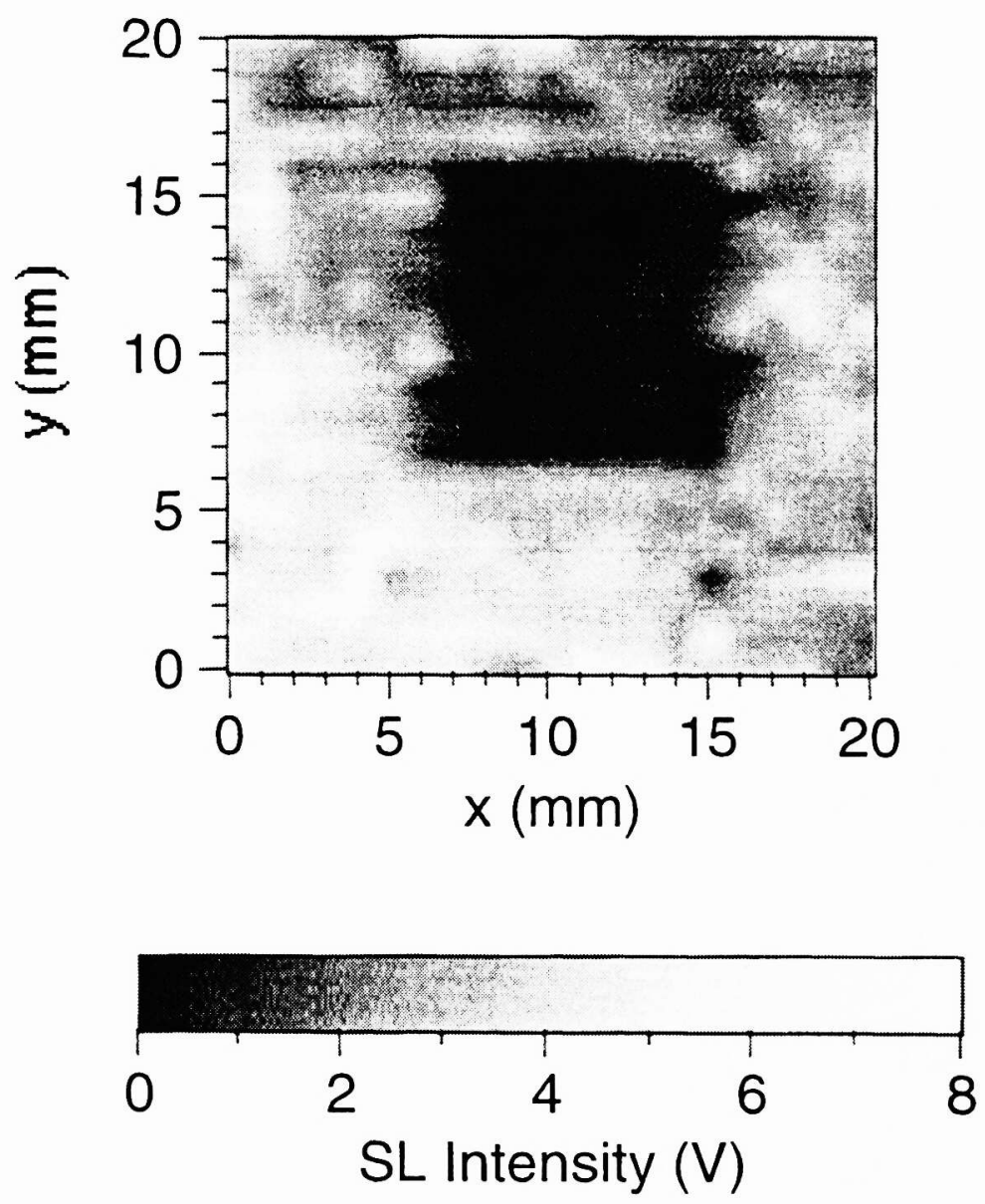

Figure 3. Two-dimensional sonoluminescence image of the object in the Intralipid phantom.

The contrast of the SL images was based on the difference between the optical and ultrasonic properties of the objects and those of the surrounding medium. These objects were optically opaque and ultrasonically absorbing. When the object was moved toward the ultrasonic focus, the SL intensity dropped quickly for several reasons. First, the ultrasonic field was reduced below the focus because of the slight acoustic attenuation of the rubber object. Second, the object yielded no SL signal. Third, the SL signal above the object was partially blocked by the object and hence was more difficult to reach the PMT.

To observe the spatial resolution for distinguishing multiple objects, we buried two cubic objects in the Intralipid phantom [Figure 4(a)]. The distance between the two objects was $\Delta x$, which was varied from $1 \mathrm{~mm}$ to $6 \mathrm{~mm}$. Onedimensional SL images across the centers of the two objects were obtained for various distances $\Delta x$ [Figure 4(b)]. From these figures, we observed the spatial resolution of $2-3 \mathrm{~mm}$, which was similar to that observed in the single-object SL images. The spatial resolution was limited by the focal size of the ultrasonic transducer, which was $3 \mathrm{~mm}$.

To observe the spatial resolution for revealing the shapes of buried objects, we buried two objects of different shapes in the Intralipid phantom: a square and a triangular object [Figure 5(a)]. A two-dimensional SL image of the two objects was acquired [Figure 5(b)]. The 2-mm separation between the tip of the triangular object and the square object was barely resolved in the SL image. The hypotenuse of the triangular object looked zigzagged because of the 1-mm step size in the raster scanning during the data acquisition. However, both objects were clearly imaged with the correct shapes and sizes. 


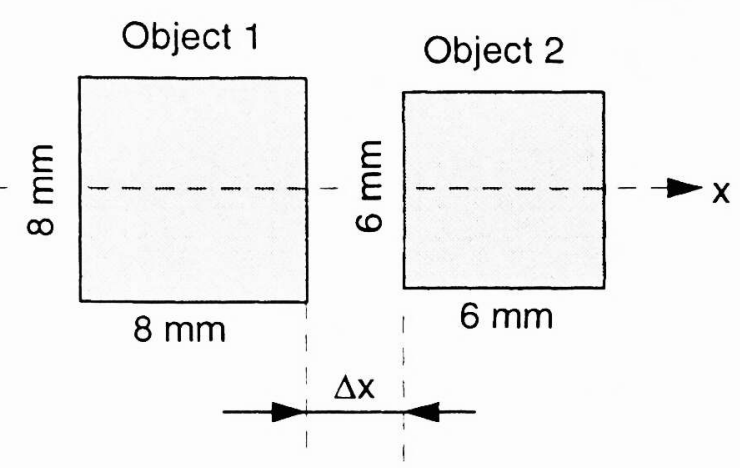

(a)

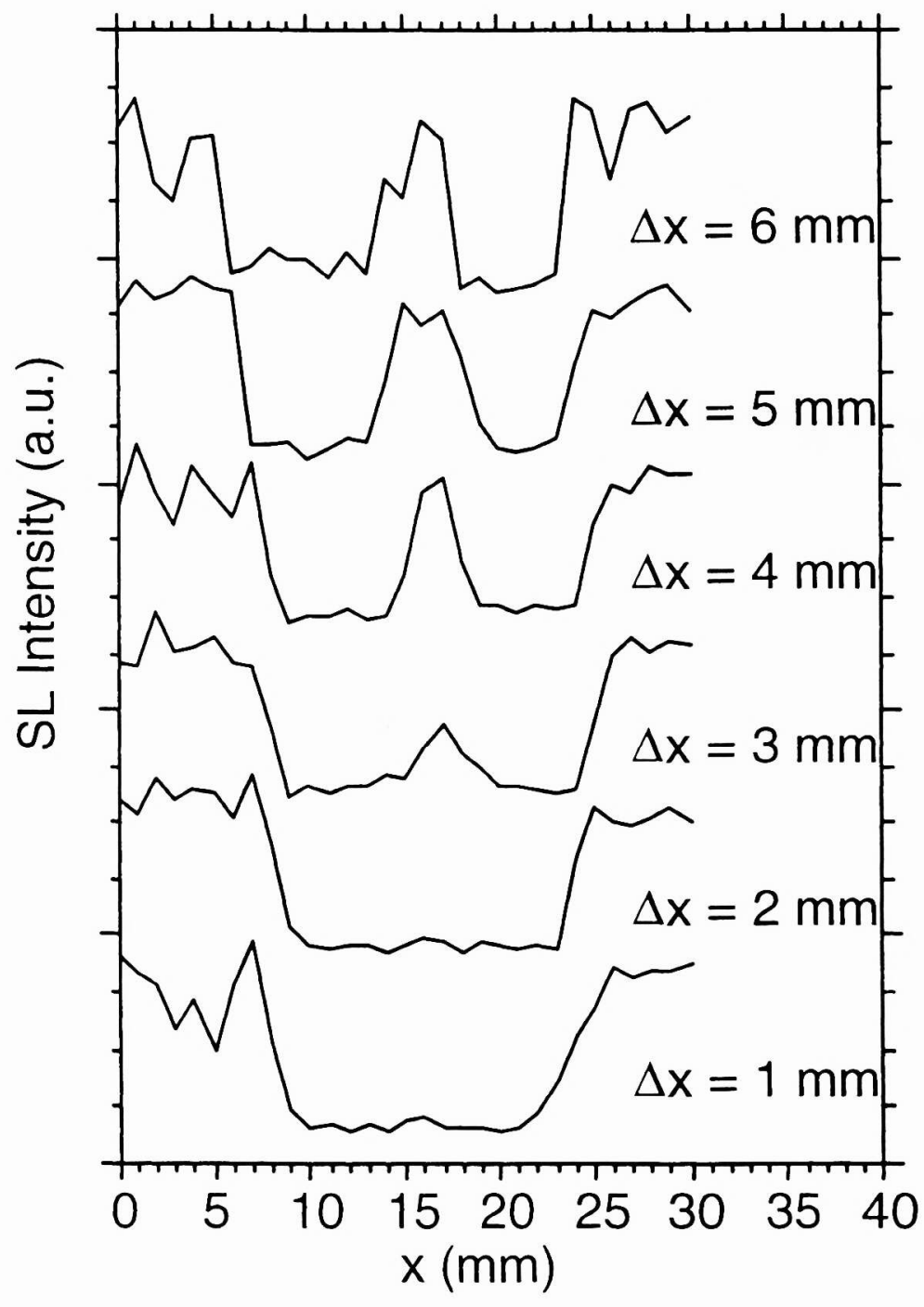

(b)

Figure 4. (a) Schematic diagram of two rubber objects buried in the Intralipid phantom with a separation $\Delta x$; (b) Onedimensional sonoluminescence images of the two objects along the $x$ axis when the separation $\Delta x$ was varied from $1 \mathrm{~mm}$ to 6 $\mathrm{mm}$. 


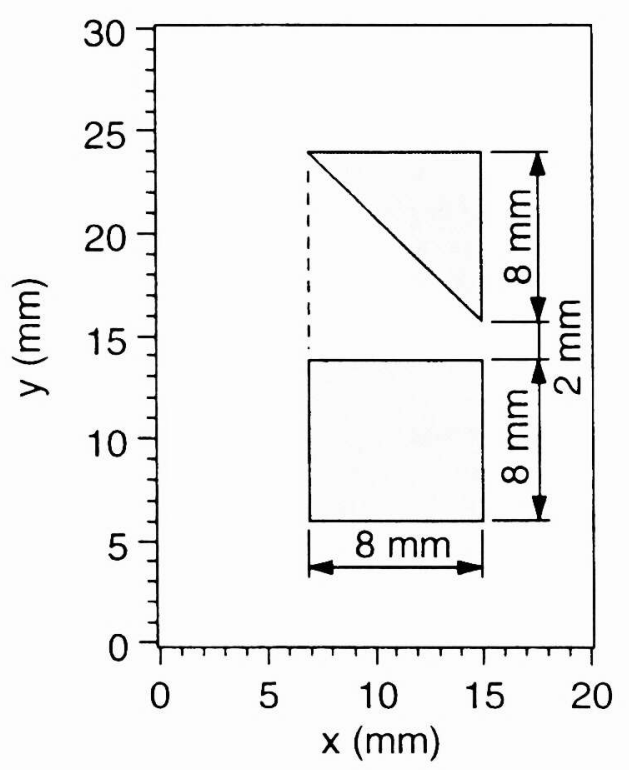

(a)
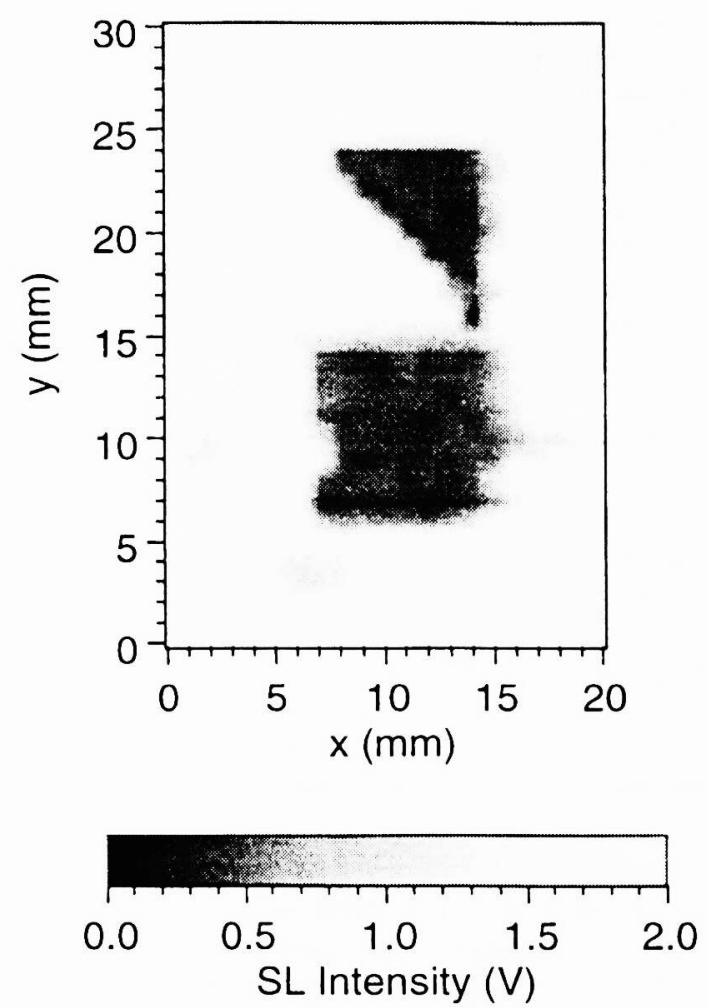

(b)

Figure 5. (a) Schematic diagram of two rubber objects with different shapes buried in the Intralipid phantom; (b) Twodimensional sonoluminescence image of the two differently shaped objects buried in the Intralipid phantom.

Although the objects used in this experiment had both optical and ultrasonic contrast relative to the background medium, images of objects may be obtained using SLT based on several contrast mechanisms in general. First, when an object has ultrasonic contrast relative to the background, the SL signal originating from the object will differ from that originating from the background medium. The SL generation is affected by the local ultrasonic intensity. Second, when an object has contrast in optical properties, the SL signal from the object will be attenuated differently because the SL light must propagate through the object. Third, when an object has contrast in ability to generate SL, the SL signal from the object will be different even if the local ultrasonic pressure is the same.

Using the present ultrasonic system, we obtained a SL column of $\sim 3.5 \mathrm{~cm}$ in length and $\sim 0.3 \mathrm{~cm}$ in diameter [Figure $2(a)] .{ }^{14}$ The length of the SL column limits the imaging resolution along the ultrasonic axis. Similarly, the diameter of the SL column determines the imaging resolution on the $x-y$ plane. A more tightly focused ultrasonic transducer may be used to reduce the size of the SL column significantly. When the SL column is reduced to a desired size, one may acquire threedimensional images of scattering media by scanning in all three directions.

SL light propagates outward in the scattering medium in all directions. We may improve the signal-noise ratio of the detection system dramatically by integrating the SL light over a large detection area or by a light collection system similar to an integrating sphere. Because all the SL light is useful for imaging, integrating the SL signal would allow an increased imaging depth as well.

Although there are potentially harmful effects caused by cavitation, the threshold of ultrasound intensity leading to volume lesions is very high. The damage threshold in spatial-peak-temporal-peak (SPTP) power was reported to be 400 $\mathrm{W} / \mathrm{cm}^{2}$ and $900 \mathrm{~W} / \mathrm{cm}^{2}$ at $1 \mathrm{MHz}$ for dog brain tissue and $\operatorname{dog}$ thigh muscle, respectively. ${ }^{19}$ The peak pressure in our experiment was -2.0 bars at the ultrasonic focus, corresponding to an SPTP power of $1.3 \mathrm{~W} / \mathrm{cm}^{2}$, which was two orders of magnitude less than the damage threshold. The peak pressure was also far less than the 23-bar safety limit set by the U.S. Food and Drug Administration, which is usually conservative. ${ }^{20}$ SLT uses ultrasonic waves to drive pre-existing microbubbles between $5 \mu \mathrm{m}$ to $\sim 50 \mu \mathrm{m}$ in size; hence, formation of new bubbles with ultrasound is not necessary. The 
threshold acoustic power to generate SL through pre-existing bubbles is much less than that required to form new bubbles. It was also reported that ultrasonic irradiation on cells using acoustic pressures less than a critical value does not cause damage, even if cavitation was observed. ${ }^{21}$ Therefore, there should be a safety window within which SL can be observed without causing tissue damage. However, careful experimental studies need to be conducted to identify the damage threshold.

In conclusion, sonoluminescence technique was used to image objects buried in dense tissue-simulating turbid media. Both the spatial resolution and the contrast of the images were very good. The spatial resolution of 2-3 mm was limited by the ultrasound focal size and may be improved by tightening the focal size. SLT should be capable of imaging thick biological tissues because of the minimal ultrasonic scattering and the broad SL spectrum. The SLT technique is expected to find applications in biomedicine and other fields involving scattering media such as clouds, ocean water, foams, paper, colloids, and dairy products. For more information regarding this work, refer to reference ${ }^{22}$.

\section{FREQUENCY-SWEPT ULTRASOUND-MODULATED OPTICAL TOMOGRAPHY}

Hybrid techniques that combine a relatively transparent radiation with the strongly scattering optical radiation are being actively developed. ${ }^{23-27}$ We report a novel imaging technique: frequency-swept ultrasound-modulated optical tomography of scattering media. In this technique a frequency-swept (chirped) ultrasonic wave was used to encode the transmitted laser light. Decoding the transmitted light in the frequency domain allowed us to image objects buried inside the scattering media. A $1 D$ image along the ultrasonic axis was obtained with a single time-domain signal.

A block diagram of the experimental setup is shown in Figure 6. A frequency-swept signal was produced using a function generator and then was amplified in power and amplitude by a power amplifier and a transformer, respectively. The instantaneous frequency of the frequency sweep was

$$
f_{s}(t)=a_{s}+b t
$$

where $a_{s}$ was the starting frequency, $b$ was the sweep rate, and $t$ was the time. In our experiment the frequency sweep was between $7.0 \mathrm{MHz}$ and $10.0 \mathrm{MHz}$ with a sweep rate of $297 \mathrm{MHz} / \mathrm{s}$. The amplified signal was applied to an ultrasonic transducer. The ultrasonic wave propagated vertically into a scattering medium, which was contained in a glass tank. An ultrasound absorber was placed at the bottom of the tank to avoid ultrasonic reflection from the water-glass interface.

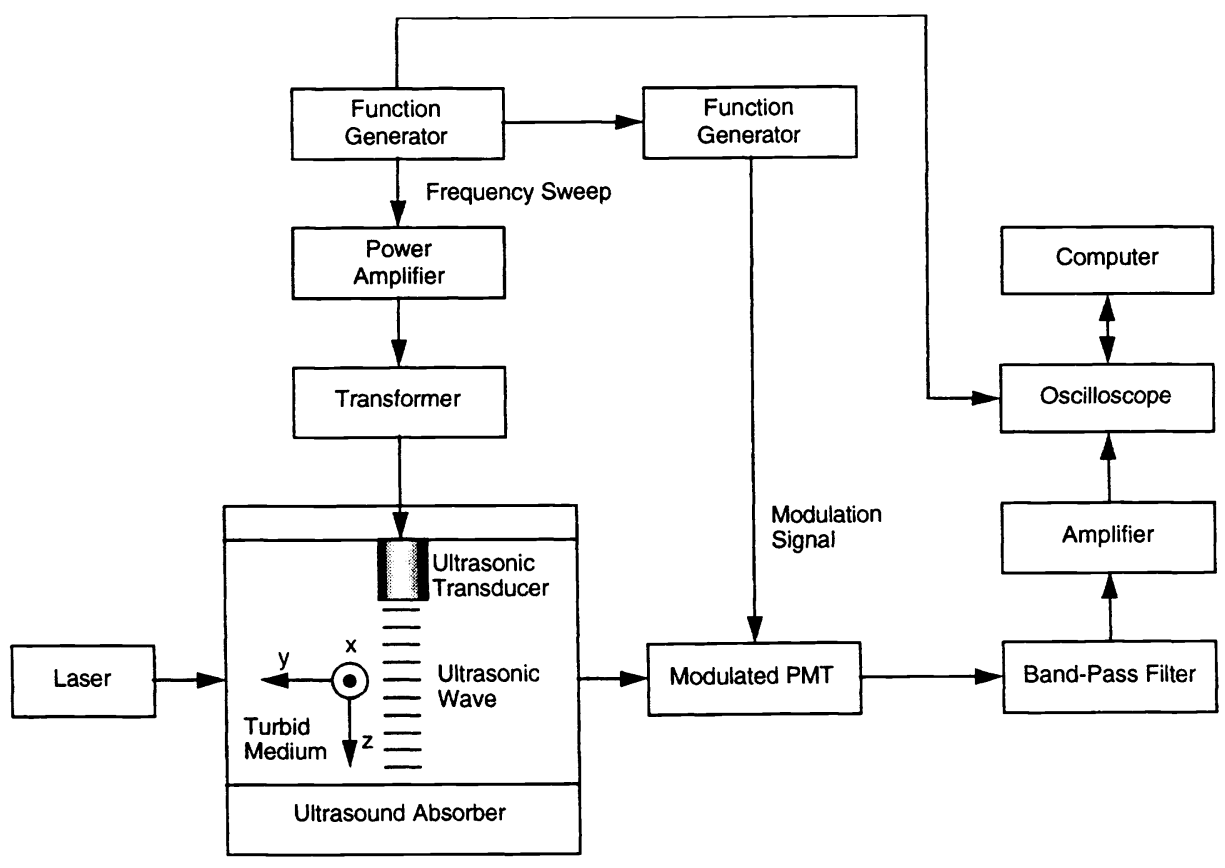

Figure 6. A block diagram of the experimental setup. 
A He-Ne laser beam at the $633 \mathrm{~nm}$ wavelength, after being broadened to $15 \mathrm{~mm}$, illuminated the scattering medium perpendicularly to the ultrasonic column. The laser light was modulated by the ultrasonic column. The instantaneous frequency of ultrasonic modulation along the column was

$$
f_{s}(t, z)=a_{s}+b\left[t-\frac{z-z_{0}}{v_{s}}\right]
$$

where $z$ was the ultrasonic axis, $z_{0}$ was the $z$ coordinate of the point of ultrasonic emission in the ultrasonic transducer, and $v_{s}$ was the speed of sound in the medium. Equation (3) was valid for $t>\left(z-z_{0}\right) / v_{s}$. A photomultiplier tube (PMT) collected some transmitted light and converted the optical power into an electric signal. The gain of the PMT was modulated for heterodyne detection by a reference modulation signal produced from another function generator. The reference modulation signal was also frequency-swept and had an instantaneous frequency

$$
f_{r}(t)=a_{r}+b t
$$

where the starting frequency $a_{r}$ of the reference modulation signal was different from that of the signal for the ultrasonic transducer.

The frequency of the heterodyned signal contributed from the ultrasonic modulation at $z$ was

$$
f_{h}(t, z)=\left|f_{s}(t, z)-f_{r}(t)\right|=\left|a_{s}-a_{r}-\frac{b\left(z-z_{0}\right)}{v_{s}}\right|,
$$

which was independent of the time $t$.

The electronically heterodyned signal at the output of the PMT was band-pass filtered and was then amplified by use of a filter and an amplifier, respectively. The band width of the filter was determined by a desired range of $z$ to be imaged (zone of detection) as follows.

$$
\Delta f_{h}=\frac{b}{v_{s}} \Delta z,
$$

where $\Delta z$ was the range of $z$ to be detected. The amplified signal was recorded by a digital oscilloscope and was then transferred to a computer for post-processing.

An object made of rubber was buried in the middle plane of the tank. The thickness of the tank along the laser beam was $17 \mathrm{~cm}$. The object was translated in the tank along the $x$ axis perpendicularly to both the laser beam and the ultrasonic column. A time-domain signal was recorded at each stop. Fast Fourier Transform (FFT) was used to compute the spectra of the recorded time-domain signals on a computer.

The frequency spectra yielded imaging information for the zone of detection. A frequency in the spectra corresponded to the difference between the instantaneous ultrasonic frequency at an ultrasonic axial position and the instantaneous frequency of the reference modulation signal [Eq. (5)]. By use of Eq. (5) the frequency spectra were converted into $1 \mathrm{D}$ images of the scattering medium along the ultrasonic axis ( $z$ axis). The imaging contrast was caused by spatial variation in the optical and acoustic properties.

Multiple 1D spectra were acquired while the buried object was scanned horizontally along the $x$ axis with a step size of $1 \mathrm{~mm}$. The first spectrum, which was taken when the object was far from the ultrasonic axis, was used as a reference spectrum. The spectral intensity of the subsequent spectra was divided by that of the reference spectrum to obtain a relative spectral intensity. A 2D image of the medium was formed by combining the 1D relative spectra at the various $x$ positions.

A 2D image of the object buried in a scattering medium is shown in Figure 7. The corresponding 1D images in both the $x$ and the $z$ directions were examined in detail (not shown) and showed that the resolution in both the $x$ and the $z$ axes was approximately $1 \mathrm{~mm}$. The scattering coefficient and anisotropy of the scattering medium were $0.21 \mathrm{~cm}^{-1}$ and 0.73 , respectively. The $z$-axis resolution was limited by the ultrasonic sweep parameters, and the $x$-axis resolution was limited by the ultrasonic focal diameter $(1.2 \mathrm{~mm})$. 

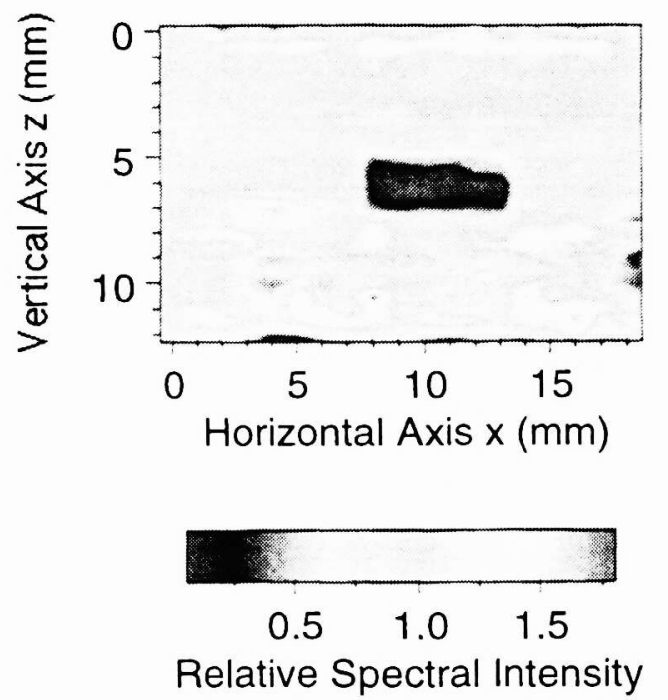

Figure 7.2D tomographic image of the object buried in a scattering medium. The object was $6 \mathrm{~mm} \times 2 \mathrm{~mm}$ in size.

This technique is currently limited to ballistic imaging and is being extended for diffuse light imaging. Because ultrasonic modulation of diffuse light with single-frequency ultrasound has been observed, ${ }^{28,29,24,25}$ ultrasonic modulation of diffuse light by use of frequency-swept ultrasound should be detectable as well. The current detection system is being improved to observe ultrasonic modulation of diffuse light by frequency-swept ultrasound. The signal-to-noise ratio of the system can not be improved by arbitrarily narrowing the band width of the detection system because the band width was proportional to the sweep rate of the frequency sweep and the zone length of detection as indicated in Eq. (6). However, a slow frequency sweep and a modest zone of detection may be used to narrow the band width. Note that the speed of sound is relatively constant in a medium and can not be varied to improve the signal-to-noise ratio.

In summary, we have experimentally proved the concept of frequency-swept ultrasound-modulated optical tomography. A ID image of a scattering medium along the ultrasonic axis may be obtained with a single time-domain signal. Therefore the imaging acquisition time can be shortened significantly compared with the single-frequency ultrasoundmodulated optical tomography. The imaging resolution is adjustable with the bandwidth of the frequency sweep and was approximately $1 \mathrm{~mm}$ in our experiment. For more information, refer to reference ${ }^{30}$.

\section{ACKNOWLEDGMENT}

Thanks to X. Zhao for her experimental assistance in electronics. This project was sponsored by the National Institutes of Health grants (R29 CA68562 and R01 CA71980) and by the National Science Foundation grant (BES-9734491).

\section{REFERENCES}

1. R. R. Alfano and J. G. Fujimoto, editors, Advances in Optical Imaging and Photon Migration, TOPS Vol. 2 (Optical Society of America, Washington, D. C., 1996).

2. B. Chance and R. R. Alfano, eds., Optical Tomography and Spectroscopy of Tissue: Theory, Instrumentation, Model, and Human Studies II, Proc. Soc. of Photo-Opt. Instrum. Eng. 2979 (SPIE Press, Bellingham, WA, 1997).

3. L.-H. Wang and S. L. Jacques, "Application of probability of $\mathrm{n}$ scatterings of light passing through an idealized tissue slab in breast imaging," in Proc. of Advances in Optical Imaging and Photon Migration, R. R. Alfano, eds., 21, 181-186 (Optical Society of America, Washington, D. C., 1994).

4. H. Frenzel and H. Schultes, "Luminescenz im ultraschallbeschickten Wasser", Z. Phys. Chem. B 27, 421-424 (1934).

5. B. P. Barber and S. J. Putterman, "Observation of synchronous picosecond sonoluminescence", Nature 352, 318-320 (1991). 

(1991).

7. $\quad$ L. A. Crum and S. Putterman, "Sonoluminescence", J. Acoust. Soc. Am. 91, 517 (1992).

8. C. C. Wu and P. H. Roberts, "Shock-wave propagation in a soncluminescencing gas bubble", Phys. Rev. Lett. 70, 3424-3427 (1993).

9. W. C. Moss, D. B. Clarke, J. W. White, and D. A. Young, "Hydrodynamic simulations of bubble collapse and picosecond sonoluminescence", Phys. Fluids 6, 2979-2985 (1994).

10. L. A. Crum and R. A. Roy, "Sonoluminescence", Science 266, 233-234 (1994).

11. C. Eberlein, "Sonoluminescence as quantum vacuum radiation", Phys. Rev. Lett. 76, 3842-3845 (1996).

12. J. B. Young, T. Schmiedel and W. Kang, "Sonoluminescence in high magnetic fields", Phys. Rev. Lett. 77, 48164819 (1996).

13. B. P. Barber, R. A. Hiller, R. Löfstedt, S. J. Putterman, and K. R. Weninger, "Defining the unknowns of sonoluminescence", Phys. Reports 281, 65-143 (1997).

14. L.-H. V. Wang and Q. Shen, "Sonoluminescent tomography of strongly scattering media", Opt. Lett. 23, 561-563 (1998).

15. R. A. Hiller and B. P. Barber, "Producing light from a bubble of air", Scientific American 272, 96 (1995).

16. L. A. Crum, "Sonoluminescence, sonochemistry, and sonophysics" J. Acoust. Soc. Am. 95, 559-562 (1994).

17. Y. T. Didenko, T. V. Gordeychuk and V. L. Koretz, "The effect of ultrasound power on water sonoluminescence", J. Sound and Vibration 147, 409-416 (1991).

18. H. J. van Staveren, C. J. M. Moes, J. van Marie, S. A. Prahl, and M. J. C. van Gemert, "Light scattering in Intralipid$10 \%$ in the wavelength range of 400-1100 nm", Appl. Opt. 30, 4507-4514 (1991).

19. F. J. Fry, N. T. Sanghvi, R. S. Foster, R. Bihrle, and C. Hennige, "Ultrasound and microbubbles: their generation, detection and potential utilization in tissue and organ therapy - experimental", Ultrasound Med. Biol. 21, 12271237 (1995).

20. T. A. Whittingham, "The safety of ultrasound", Imaging 6, 33-51 (1994).

21. S. Daniels, T. Kodama and D. J. Price, "Damage to red blood cells induced by acoustic cavitation", Ultrasound Med. Biol. 21, 105-111 (1995).

22. Q. Shen and L.-H. Wang, "Two-dimensional imaging of dense tissue-simulating turbid media by use of sonoluminescence," Applied Optics 38, 246 - 252 (1999).

23. L.-H. Wang, S. L. Jacques, and X.-M. Zhao, "Continuous-wave ultrasonic modulation of scattered laser light to image objects in turbid media," Optics Letters 20, 629-631 (1995).

24. M. Kempe, M. Larionov, D. Zaslavsky, and A. Z. Genack, "Acousto-optic tomography with multiply-scattered light," J. Opt. Soc. Am. 4, 1151-1158 (1997).

25. L.-H. Wang and X.-M. Zhao, "Ultrasound-modulated optical tomography of absorbing objects buried in dense tissue-simulating turbid media," Applied Optics 36, 7277-7282 (1997).

26. A. A. Oraevsky, R. Esenaliev, F. K. Tittel, M. R. Ostermeyer, L.-H. Wang, and S. L. Jacques, "Laser opto-acoustic imaging of turbid media: determination of optical properties by comparison with diffusion theory and Monte Carlo simulation," in Laser-Tissue Interaction VII, S. L. Jacques, eds., Proc. Soc. Photo-Opt. Instrum. Eng. 2681, 277-284 (SPIE Press, 1996).

27. R. A. Kruger, P. Liu, Y. R. Fang, and C. R. Appledorn, "Photoacoustic ultrasound (PAUS)--reconstruction tomography," Medical Physics 22, 1605-1609 (1995).

28. F. A. Marks, H. W. Tomlinson, and G. W. Brooksby, "A comprehensive approach to breast cancer detection using light: photon localization by ultrasound modulation and tissue characterization by spectral discrimination," in Proc. Soc. Photo-Opt. Instrum. Eng., eds., 1888, 500-510 (1993).

29. W. Leutz and G. Maret, "Ultrasonic modulation of multiply scattered light," Physica B 204, 14-19 (1995).

30. L.-H. Wang and G. Ku, "Frequency-swept ultrasound-modulated optical tomography of scattering media," Optics Letters 23, 975-977 (1998). 